BASSET: Scalable Gateway Finder in Large Graphs

H. Tong, S. Papadimitriou, C. Faloutsos, P. S. Yu,

T. Eliassi-Rad

November 4, 2010

Pacific-Asia Conference on Knowledge Discovery and Data Mining

Hyderabad, India

June 21, 2010 through June 24, 2010 
This document was prepared as an account of work sponsored by an agency of the United States government. Neither the United States government nor Lawrence Livermore National Security, LLC, nor any of their employees makes any warranty, expressed or implied, or assumes any legal liability or responsibility for the accuracy, completeness, or usefulness of any information, apparatus, product, or process disclosed, or represents that its use would not infringe privately owned rights. Reference herein to any specific commercial product, process, or service by trade name, trademark, manufacturer, or otherwise does not necessarily constitute or imply its endorsement, recommendation, or favoring by the United States government or Lawrence Livermore National Security, LLC. The views and opinions of authors expressed herein do not necessarily state or reflect those of the United States government or Lawrence Livermore National Security, LLC, and shall not be used for advertising or product endorsement purposes. 


\title{
BASSET: Scalable Gateway Finder in Large Graphs
}

\author{
Hanghang Tong* $\quad$ Spiros Papadimitriou ${ }^{\dagger} \quad$ Christos Faloutsos* \\ Philip S. Yu ${ }^{\ddagger} \quad$ Tina Eliassi-Rad ${ }^{\S}$
}

\begin{abstract}
Given a social network, who is the best person to introduce you to, say, Chris Ferguson, the poker champion? Or, given a network of people and skills, who is the best person to help you learn about, say, wavelets? The goal is to find a small group of 'gateways': persons who are close enough to us, as well as close enough to the target (person, or skill) or, in other words, are crucial in connecting us to the target.

The main contributions are the following: (a) we show how to formulate this problem precisely; (b) we show that it is sub-modular and thus it can be solved near-optimally; (c) we give fast, scalable algorithms to find such gateways. Experiments on real data sets validate the effectiveness and efficiency of the proposed methods, achieving up to $6,000,000 \times$ speedup.
\end{abstract}

\section{Introduction}

What is the best gateway between a source node and a target node, in a network? This is a core problem that appears under several guises, with numerous generalizations. Motivating applications include the following:

1. In a corporate social network, which are the key people that bring or hold different groups together? Or, if seeking to establish a cross-division project, who are the best people to lead such an effort?

2. In an immunization setting, given a set of nodes that are infected, and a set of nodes we want to defend, which are the best few 'gateways' we should immunize?

3. Similarly, in a network setting, which are the gateway nodes we should best defend against an attack, to maximize connectivity from source to target.

4. Protein pathways: given a protein interaction network, we know that a certain protein group corresponds to an earlier type of flu (e.g., normal flu), and another group corresponds to a new type of flu (e.g., swine flu), and we want to know which other proteins play a critical role in developing normal flu towards swine flu.

5. Given a graph of co-workers and their skills (keywords), whom should you contact to learn more about, say, Linux? You want someone reasonably close to you and fairly well-versed in Linux, but not your secretary or Linus Torvalds himself.

The problem has several, natural generalizations: (a) we may be interested in the top $k$ best gateways (in case our first few choices are unavailable); (b) we may have more than one source nodes, and more than one target nodes, as in the immunization setting above; (c) we may have a bi-partite graph with relationships (edges) between different node types, as in the last example above. Our main contributions in this paper are:

- A novel 'gateway-ness' score for a given source and target, that agrees with human intuition. Its generalization to the case where we have a group of nodes as the source and the target;

\footnotetext{
*Carnegie Mellon University, $\{$ htong, christos\}@cs.cmu.edu

† IBM T.J. Watson, spapadim@us.ibm.com

${ }_{\ddagger}$ University of Illinois at Chicago, psyu@cs.uic.edu

§Lawrence Livermore National Laboratory, eliassi@1lnl.gov
} 
- Two algorithms to find a set of nodes with the highest 'gateway-ness' score, which (1) are fast and scalable; and (2) lead to near-optimal results;

- Extensive experimental results on real data sets, showing the effectiveness and efficiency of the proposed methods.

The rest of the paper is organized as follows: We give the problem definitions in Section 2; present 'gateway-ness' scores in Section 3; and deal with the computational issues in Section 4. We evaluate the proposed methods in Section 5. Finally, we review the related work in Section 6 and conclude in Section 7.

\section{Problem Definitions}

Table 1: Symbols

\begin{tabular}{|l|l|}
\hline Symbol & Definition and Description \\
\hline \hline $\mathbf{A}, \mathbf{B}, \ldots$ & matrices (bold upper case) \\
$\mathbf{A}(i, j)$ & the element at the $i^{t h}$ row and $j^{t h}$ column of matrix $\mathbf{A}$ \\
$\mathbf{A}(i,:)$ & the $i^{t h}$ row of matrix $\mathbf{A}$ \\
$\mathbf{A}(:, j)$ & the $j^{t h}$ column of matrix $\mathbf{A}$ \\
$\mathbf{A}^{\prime}$ & transpose of matrix $\mathbf{A}$ \\
$\mathbf{a}, \mathbf{b}, \ldots$ & column vectors \\
$\vec{p}, \vec{q}, \ldots$ & ordered sequences \\
$\mathcal{S}, \mathcal{T}, \ldots$ & sets (calligraphic) \\
\hline$n$ & number of nodes in the graph \\
$m$ & number of edges in the graph \\
\hline $\mathrm{g}(s, t, \mathcal{I})$ & the 'Gateway-ness' score for the subset of nodes $\mathcal{I}$ wrt the source $s$ and the target $t$ \\
$\mathrm{~g}(\mathcal{S}, \mathcal{T}, \mathcal{I})$ & the 'Gateway-ness' score for the subset of nodes $\mathcal{I}$ wrt the source group $\mathcal{S}$ and the target group $\mathcal{T}$ \\
$\mathbf{r}(s, t)$ & the proximity score from $s$ to $t$ \\
$\mathbf{r}_{\mathcal{I}}(s, t)$ & the proximity score from $s$ to $t$ by setting the subset of nodes indexed by $\mathcal{I}$ as sinks \\
\hline
\end{tabular}

Table 1 lists the main symbols we use throughout the paper. In this paper, we focus on directed weighted graphs. We represent the graph by its normalized adjacency matrix (A). Following standard notation, we use capital bold letters for matrices (e.g., A), lower-case bold letters for vectors (e.g., a), and calligraphic fonts for sets (e.g., $\mathcal{S}$ ). We denote the transpose with a prime (i.e., $\mathbf{A}^{\prime}$ is the transpose of $\mathbf{A}$ ). We use arrowed lower-case letters for paths on the graph (e.g., $\vec{p}$ ), which are ordered sequences. We use parenthesized superscripts to represent source/target information for the corresponding variables. For example $\vec{p}^{(s, t)}=\left\{s=u_{0}, u_{1}, \ldots, u_{l}=t\right\}$ is a path from the source node $s$ to the target node $t$. If the source/target information is clear from the context, we omit the superscript for brevity. A sink node $i$ on the graph is a node without out-links (i.e., $\mathbf{A}(:, i)=0$ ). We use subscripts to denote the corresponding variable after setting the nodes indexed by the subscripts as sinks. For example, $\vec{p}_{\mathcal{I}}^{(s, t)}$ is the path from the source node $s$ to the target node $t$, which does not go through any nodes indexed by the set $\mathcal{I}$ (i.e., $u_{i} \notin \mathcal{I}, i=0, \ldots, l$ ). With the above notations, our problems can be formally defined as follows:

\section{Problem 1 (Pair-Gateway)}

Given: a weighted directed graph $\mathbf{A}$, a source node s, a target node $t$, and a budget (integer) $k$;

Find: a set of at most $k$ nodes which has the highest 'gate-way-ness' score wrt $s$ and $t$.

\section{Problem 2 (Group-Gateway)}

Given: a weighted directed graph $\mathbf{A}$, a group of source nodes $\mathcal{S}$, a group of target nodes $\mathcal{T}$, and a budget (integer) $k$; Find: a set of at most $k$ nodes which has the highest 'gate-way-ness' score wrt $\mathcal{S}$ and $\mathcal{T}$.

In both Problem 1 (Pair-Gateway) and Problem 2 (Group-Gateway), there are two sub-problems: (1) how to define the 'gateway-ness' score of a given subset of nodes $\mathcal{I}$; (2) how to find the subset of nodes with the highest 'gateway-ness' score. In the next two sections, we present the solutions for each, respectively. 


\section{Proposed 'Gateway-ness' Scores}

In this section, we present our definitions for 'Gateway-ness'. We first focus on the case of a single source $s$ and a single target $t$ (Pair-Gateway). We then generalize to the case where both the source and the target are a group of nodes (Group-Gateway)

\subsection{Node 'Gateway-ness' Score}

Given a single source $s$ and a single target $t$, we want to measure the 'Gateway-ness' score for a given set of nodes $\mathcal{I}$. We first give the formal definitions in such a setting and then provide some intuitions for our definitions.

Formal Definitions. For a graph A, we can use random walk with restart to measure the proximity (i.e., relevance/closeness) from the source node $s$ to the target node $t$, which is defined as follows: Consider a random particle that starts from node $s$. The particle iteratively transits to its neighbors with probability proportional to the corresponding edge weights. Also at each step, the particle returns to node $s$ with some restart probability $(1-c)$. The proximity score from node $s$ to node $t$ is defined as the steady-state probability $\mathbf{r}(s, t)$ that the particle will be on node $t$ [30]. Intuitively, $\mathbf{r}(s, t)$ is the fraction of time that the particle starting from node $s$ will spend on node $t$ of the graph, after an infinite number of steps.

Intuitively, a set of nodes $\mathcal{I}$ are good gateways wrt $s$ and $t$ if they play an important role in the proximity measure from the source to the target. Therefore, our 'Gateway-ness' score can be defined as follows:

$$
\mathrm{g}(s, t, \mathcal{I}) \triangleq \Delta \mathbf{r}(s, t) \triangleq \mathbf{r}(s, t)-\mathbf{r}_{\mathcal{I}}(s, t)
$$

where $r_{\mathcal{I}}(s, t)$ is the proximity score from source $s$ to $t$ after setting the subset of nodes indexed by $\mathcal{I}$ as sinks.

Intuitions. Here, we provide some intuition of the 'Gateway-ness' score defined by eq.(1), using the running example in figure 1.

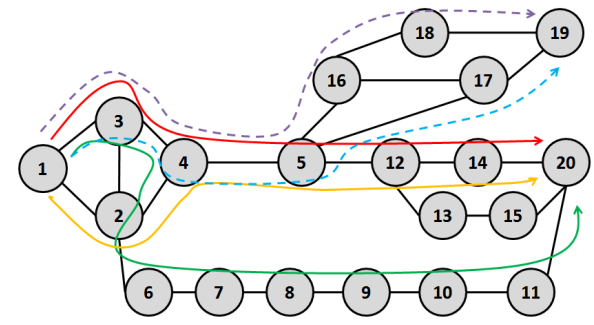

Figure 1: Running example (best viewed in color)

In figure 1, each solid arrowed line is a path from node 1 to node 20 , which can be denoted by an ordered sequence. For example, the path marked by the red line can be denoted by $\vec{p}^{(1,20)}=\{1,3,4,5,12,14,20\}$. For each path $\vec{p}^{(s, t)}=\left\{s=u_{0}, u_{1}, \ldots, u_{l}=t\right\}$, we can define its score by eq (2), where $\prod_{i=0}^{l} \mathbf{A}\left(u_{i-1}, u_{i}\right)$ is the probability that the random particle will traverse this path, and $(1-c) c^{l}$ penalizes the length of the path. For example, the red path $\left(\vec{p}^{(1,20)}=\{1,3,4,5,12,14,20\}\right)$, has score $(1-c) c^{6} \mathbf{A}(3,1) \mathbf{A}(4,3) \mathbf{A}(5,4) \mathbf{A}(12,5) \mathbf{A}(14,12) \mathbf{A}(20,14)$.

$$
\operatorname{score}\left(\vec{p}^{(s, t)}\right) \triangleq(1-c) c^{l} \prod_{i=0}^{l} \mathbf{A}\left(u_{i-1}, u_{i}\right)
$$

where $\mathbf{A}$ is the normalized adjacency matrix of the graph.

With the above definitions for the path score, we have the following lemma: 
Lemma 1 Sum of Weighted Path Scores. Let $\vec{P}$ be the set of all the paths from the source node s to the target node $t$, and $\vec{Q}$ be the set of all the paths from the source node $s$ to the target node $t$ which go through at least one node indexed by the subset $\mathcal{I}$. Let $\mathbf{r}(s, t)$ be the proximity score defined by random walk with restart and $g(s, t, \mathcal{I})$ be the 'Gateway-ness' score defined by eq. (1). Then we have

$$
\mathbf{r}(s, t)=\sum_{\vec{p}^{(s, t)} \in \vec{P}} \operatorname{score}\left(\vec{p}^{(s, t)}\right) ; \quad g(s, t, \mathcal{I})=\sum_{\vec{p}^{(s, t)} \in \vec{Q}} \operatorname{score}\left(\vec{p}^{(s, t)}\right)
$$

Proof: Omitted for space.

By eq. (3), the 'Gateway-ness' score for a given set of nodes $\mathcal{I}$ accounts for all the paths from the source node $s$ to the target node $t$ which pass through one or more nodes in $\mathcal{I}$. For example, given the source node 1 and the target node 20 in figure 1, the 'Gateway-ness' score for $\mathcal{I}=\{2\}$ is the sum of the scores of all the paths from node 1 to node 20 that go through node 2 (e.g., the green path, the yellow path, and so on).

\subsection{Group 'Gateway-ness' Score}

Here we consider the case where the source and/or target consist of more than one nodes. Suppose we have a group of source nodes $\mathcal{S}$ and a group of target nodes $\mathcal{T}$. Then, the 'Gateway-ness' score for a given set of nodes $\mathcal{I}$ can be defined in a similar way:

$$
\mathrm{g}(\mathcal{S}, \mathcal{T}, \mathcal{I}) \triangleq \sum_{s \in \mathcal{S}, t \in \mathcal{T}} \Delta \mathbf{r}(s, t) \triangleq \sum_{s \in \mathcal{S}, t \in \mathcal{T}}\left(\mathbf{r}(s, t)-\mathbf{r}_{\mathcal{I}}(s, t)\right)
$$

where $r_{\mathcal{I}}(s, t)$ is the proximity score from $s$ to $t$ by setting the subset of nodes indexed by $\mathcal{I}$ as sinks (i.e., delete all out-edges, by setting $\mathbf{A}(:, i)=0$ for all $i \in \mathcal{I})$.

Intuitively, the score defined by eq. (4) accounts for all the paths from the source group to the target group ${ }^{1}$ which go through at least one node in $\mathcal{I}$. For example, given $\mathcal{S}=\{1\}$ and $\mathcal{T}=\{19,20\}$ in figure 1, the group 'Gateway-ness' score for $\mathcal{I}=\{5,8\}$ corresponds to all the paths from node 1 to 19 or 20 (e.g., red, yellow and green solid lines, purple and blue dashed lines and so on).

\section{BASSET: Proposed Fast Solutions}

In this section, we address how to quickly find a subset of nodes of the highest 'Gateway-ness' score. We start by showing that the straight-forward methods (referred to as 'Com-RWR') are computationally intractable. Then, we present the proposed BASSET (BASSET-N for Pair-Gateway and BASSET-G for Group-Gateway). For each case, we first present the algorithm and then analyze its effectiveness as well as its computational complexity.

\subsection{Computational Challenges}

Here, we present the computational challenges and the way we tackle them. For the sake of succinctness, we mainly focus on BASSET-N.

There are two main computational challenges in order to find a subset of nodes with the highest 'Gateway-ness' score. First of all, we need to compute the proximity from the source to the target on different graphs, each of which is a perturbed version of the original graph. This essentially means that we cannot directly apply some powerful pre-computational method to evaluate the proximity from the source to the target (after setting the subset of nodes indexed by $\mathcal{I}$ as sinks). Instead, we have to rely on on-line iterative methods, whose computational complexity is $O(m)$. The challenges are compounded by the need to evaluate $\mathrm{g}(s, t, \mathcal{I})$ (eq. (1)) or $\mathrm{g}(\mathcal{S}, \mathcal{T}, \mathcal{I})$ (eq. (4)) an exponential number of times $\left(\left(\begin{array}{l}n \\ k\end{array}\right)\right)$. Putting these together, the straightforward way to find $k$ nodes with the highest 'Gateway-ness' score is $O\left(\left(\begin{array}{l}n \\ k\end{array}\right) m\right)$. This is computationally intractable. Suppose on a graph with $1,000,000$ nodes, we want to find the best $k=5$ gateway nodes. If computing each proximity score takes 0.001 seconds, then $2.64 \times 10^{17}$ years are needed to find the gateways. This is much longer than the age of the universe. ${ }^{2}$

To tackle such challenges, we resort to two main ideas, which are summarized in Theorem 1. According to Theorem 1, in order to evaluate the 'Gateway-ness' score of a given set of nodes, we do not need to actually set these nodes as sinks and compute

\footnotetext{
${ }^{1} \mathrm{~A}$ path from the source group to the target group is a path which starts from a node of the source group and ends at a node of the target group.

${ }^{2}$ According to Wikipedia, (http: //en.wikipedia.org/wiki/ Age_of_the_universe), the age of the universe is about $1.4 \times 10^{10}$ year.
} 
the proximity score on the new graph. Instead, we can compute it from the original graph. In this way, we can utilize methods based on pre-computation to accelerate the process. Furthermore, since $\mathrm{g}(s, t, \mathcal{I})$ and $\mathrm{g}(\mathcal{S}, \mathcal{T}, \mathcal{I})$ are sub-modular wrt $\mathcal{I}$, we can develop some greedy algorithm to avoid exponential enumeration, and still get some near-optimal solution. In Theorem 1, A is the normalized adjacency matrix of the graph. It is worth pointing out that The proposed methods (BASSET-N and BASSET-G) we will introduce are orthogonal to the specific way of normalization. For simplicity, we use column-normalization throughout this paper. Also, $\mathbf{Q}(\mathcal{I}, \mathcal{I})$ is a $|\mathcal{I}| \times|\mathcal{I}|$ matrix, containing the elements in the matrix $\mathbf{Q}$ which are at the rows/columns indexed by $\mathcal{I}$. Similarly, $\mathbf{Q}(t, \mathcal{I})$ is a row vector with length $|\mathcal{I}|$, containing the elements in the matrix $\mathbf{Q}$ which are at the $t^{\text {th }}$ row and the columns indexed by $\mathcal{I} . \mathbf{Q}(\mathcal{I}, s)$ is a column vector with length $|\mathcal{I}|$, containing the elements in the matrix $\mathbf{Q}$ which are at the $s^{\text {th }}$ column and the rows indexed by $\mathcal{I}$.

Theorem 1 Core Theorem. Let $\mathbf{A}$ be the normalized adjacency matrix of the graph, and $\mathbf{Q}=(1-c)(\mathbf{I}-c \mathbf{A})^{-1}$. For a given source $s$ and target $t$, the 'Gateway-ness' score of a subset of nodes $\mathcal{I}$ defined in eq. (1) satisfies the properties $P 1$ and $P 2$. For a given source group $\mathcal{S}$ and target group $\mathcal{T}$, the 'Gateway-ness' score of a subset of nodes $\mathcal{I}$ defined in eq. (4) satisfies the properties $P 3$ and $P 4$, where $s \neq t, s, t \notin \mathcal{I}, \mathcal{S} \cap \mathcal{T}=\emptyset, \mathcal{S} \cap \mathcal{I}=\emptyset$, and $\mathcal{T} \cap \mathcal{I}=\emptyset$.

P1. $g(s, t, \mathcal{I})=\mathbf{Q}(t, \mathcal{I}) \mathbf{Q}(\mathcal{I}, \mathcal{I})^{-1} \mathbf{Q}(\mathcal{I}, s)$;

P2. $g(s, t, \mathcal{I})$ is sub-modular wrt the set $\mathcal{I}$.

P3. $g(\mathcal{S}, \mathcal{T}, \mathcal{I})=\sum_{s \in \mathcal{S}, t \in \mathcal{T}} \mathbf{Q}(t, \mathcal{I}) \mathbf{Q}(\mathcal{I}, \mathcal{I})^{-1} \mathbf{Q}(\mathcal{I}, s)$;

P4. $g(\mathcal{S}, \mathcal{T}, \mathcal{I})$ is sub-modular wrt the $\operatorname{set} \mathcal{I}$.

Proof of P1: WLOG, we assume that $\mathcal{I}=\{n-k+1, \ldots n\}$. Let $\mathbf{A}$ and $\tilde{\mathbf{A}}$ be the normalized adjacency matrices of the graph before/after we set the subset of nodes in $\mathcal{I}$ as sinks. Write $\mathbf{A}$ and $\tilde{\mathbf{A}}$ in block form:

$$
\mathbf{A}=\left(\begin{array}{ll}
\mathbf{A}_{1,1} & \mathbf{A}_{1,2} \\
\mathbf{A}_{2,1} & \mathbf{A}_{2,2}
\end{array}\right), \tilde{\mathbf{A}}=\left(\begin{array}{cc}
\tilde{\mathbf{A}}_{1,1} & \tilde{\mathbf{A}}_{1,2} \\
\tilde{\mathbf{A}}_{2,1} & \tilde{\mathbf{A}}_{2,2}
\end{array}\right)=\left(\begin{array}{ll}
\mathbf{A}_{1,1} & \mathbf{0} \\
\mathbf{A}_{2,1} & \mathbf{0}
\end{array}\right)
$$

where $\mathbf{0}$ is a matrix with all zero elements.

Let $\tilde{\mathbf{Q}}=(1-c)(\mathbf{I}-c \tilde{\mathbf{A}})^{-1}$. We can also write $\tilde{\mathbf{Q}}$ and $\mathbf{Q}$ in block form:

$$
\begin{aligned}
& \mathbf{Q}=(1-c)(\mathbf{I}-c \mathbf{A})^{-1}=\left(\begin{array}{ll}
\mathbf{Q}_{1,1} & \mathbf{Q}_{1,2} \\
\mathbf{Q}_{2,1} & \mathbf{Q}_{2,2}
\end{array}\right)=(1-c)\left(\begin{array}{cc}
\mathbf{I}-c \mathbf{A}_{1,1} & -c \mathbf{A}_{1,2} \\
-c \mathbf{A}_{2,1} & \mathbf{I}-c \mathbf{A}_{2,2}
\end{array}\right)^{-1} \\
& \tilde{\mathbf{Q}}=\left(\begin{array}{ll}
\tilde{\mathbf{Q}}_{1,1} & \tilde{\mathbf{Q}}_{1,2} \\
\tilde{\mathbf{Q}}_{2,1} & \tilde{\mathbf{Q}}_{2,2}
\end{array}\right)=(1-c)\left(\begin{array}{cc}
\mathbf{I}-c \mathbf{A}_{1,1} & \mathbf{0} \\
-c \mathbf{A}_{2,1} & \mathbf{I}
\end{array}\right)^{-1}
\end{aligned}
$$

Applying the block matrix inverse lemma [26] to $\tilde{\mathbf{Q}}$ and $\mathbf{Q}$, we get the following equations:

$$
\begin{aligned}
& \tilde{\mathbf{Q}}_{1,1}=(1-c)\left(\mathbf{I}-c \mathbf{A}_{1,1}\right)^{-1}, \tilde{\mathbf{Q}}_{1,2}=\mathbf{0} \\
& \tilde{\mathbf{Q}}_{2,1}=c(1-c) \mathbf{A}_{2,1}\left(\mathbf{I}-c \mathbf{A}_{1,1}\right)^{-1}, \tilde{\mathbf{Q}}_{2,2}=(1-c) \mathbf{I} \\
& \mathbf{Q}_{1,1}=(1-c)\left(\mathbf{I}-c \mathbf{A}_{1,1}\right)^{-1}+c^{2}\left(\mathbf{I}-c \mathbf{A}_{1,1}\right)^{-1} \mathbf{A}_{1,2} \mathbf{Q}_{2,2} \mathbf{A}_{2,1}\left(\mathbf{I}-c \mathbf{A}_{1,1}\right)^{-1} \\
& \mathbf{Q}_{1,2}=c\left(\mathbf{I}-c \mathbf{A}_{1,1}\right)^{-1} \mathbf{A}_{1,2} \mathbf{Q}_{2,2} \\
& \mathbf{Q}_{2,1}=c \mathbf{Q}_{2,2} \mathbf{A}_{2,1}\left(\mathbf{I}-c \mathbf{A}_{1,1}\right)^{-1}
\end{aligned}
$$

Therefore, we have

$$
\tilde{\mathbf{Q}}_{1,1}=\mathbf{Q}_{1,1}-\mathbf{Q}_{1,2} \mathbf{Q}_{2,2}^{-1} \mathbf{Q}_{2,1}
$$

On the other hand, based on the properties of random walk with restart [30], we have $\mathbf{r}(i, j)=\mathbf{Q}(j, i)$, and $\mathbf{r}_{\mathcal{I}}(i, j)=$ $\tilde{\mathbf{Q}}(j, i),(i, j=1, \ldots, n)$. Together with eq. (7), we have

$$
\mathrm{g}(s, t, \mathcal{I})=\mathbf{r}(s, t)-\mathbf{r}_{\mathcal{I}}(s, t)=\mathbf{Q}_{1,1}(t, s)-\tilde{\mathbf{Q}}_{1,1}(t, s)=\mathbf{Q}_{1,2}(t,:) \mathbf{Q}_{2,2}^{-1} \mathbf{Q}_{1,2}(:, s)
$$

which completes the proofs of P1.

Proof of P3: Since P1 holds, we have

$$
\mathrm{g}(\mathcal{S}, \mathcal{T}, \mathcal{I})=\sum_{s \in \mathcal{S}, t \in \mathcal{T}} \Delta r(s, t)=\sum_{s \in \mathcal{S}, t \in \mathcal{T}} \mathrm{g}(s, t, \mathcal{I})=\sum_{s \in \mathcal{S}, t \in \mathcal{T}} \mathbf{Q}(t, \mathcal{I}) \mathbf{Q}(\mathcal{I}, \mathcal{I})^{-1} \mathbf{Q}(\mathcal{I}, s)
$$

which completes the proofs of P3. 
Proof of P2: Let $\mathcal{I}, \mathcal{J}, \mathcal{K}$ be three subsets and $\mathcal{I} \subseteq \mathcal{J}$. We will first prove by induction that, for any integer power $j$, the following inequality holds element-wise.

$$
\mathbf{A}_{\mathcal{I}}^{j}-\mathbf{A}_{\mathcal{I} \cup \mathcal{K}}^{j} \geq \mathbf{A}_{\mathcal{J}}^{j}-\mathbf{A}_{\mathcal{J} \cup \mathcal{K}}^{j}
$$

It is easy to verify the base case (i.e., $j=1$ ) for eq. (10) holds. Next, assume that eq. (10) holds for $j=1, \ldots, j_{0}$, and we want to prove that it also holds for the case $j=j_{0}+1$ :

$$
\begin{aligned}
\mathbf{A}_{\mathcal{I}}^{j_{0}+1}-\mathbf{A}_{\mathcal{I} \cup \mathcal{K}}^{j_{0}+1} & =\mathbf{A}_{\mathcal{I}}^{j_{0}+1}-\mathbf{A}_{\mathcal{I} \cup \mathcal{K}}^{j_{0}} \mathbf{A}_{\mathcal{I}}+\mathbf{A}_{\mathcal{I} \cup \mathcal{K}}^{j_{0}} \mathbf{A}_{\mathcal{I}}-\mathbf{A}_{\mathcal{I} \cup \mathcal{K}}^{j_{0}+1} \\
& =\left(\mathbf{A}_{\mathcal{I}}^{j_{0}}-\mathbf{A}_{\mathcal{I} \cup \mathcal{K}}^{j_{0}}\right) \mathbf{A}_{\mathcal{I}}+\mathbf{A}_{\mathcal{I} \cup \mathcal{K}}^{j_{0}}\left(\mathbf{A}_{\mathcal{I}}-\mathbf{A}_{\mathcal{I} \cup \mathcal{K}}\right) \\
& \geq\left(\mathbf{A}_{\mathcal{J}}^{j_{0}}-\mathbf{A}_{\mathcal{J} \cup \mathcal{K}}^{j_{0}}\right) \mathbf{A}_{\mathcal{I}}+\mathbf{A}_{\mathcal{I} \cup \mathcal{K}}^{j_{0}}\left(\mathbf{A}_{\mathcal{J}}-\mathbf{A}_{\mathcal{J} \cup \mathcal{K}}\right) \\
& \geq\left(\mathbf{A}_{\mathcal{J}}^{j_{0}}-\mathbf{A}_{\mathcal{J} \cup \mathcal{K}}^{j_{0}}\right) \mathbf{A}_{\mathcal{J}}+\mathbf{A}_{\mathcal{J} \cup \mathcal{K}}^{j_{0}}\left(\mathbf{A}_{\mathcal{J}}-\mathbf{A}_{\mathcal{J} \cup \mathcal{K}}\right)=\mathbf{A}_{\mathcal{J}}^{j_{0}+1}-\mathbf{A}_{\mathcal{J} \cup \mathcal{K}}^{j_{0}+1}
\end{aligned}
$$

In eq. (11), the first inequality holds because of the induction assumption. The second inequality holds because $\mathbf{A}_{\mathcal{I}} \geq \mathbf{A}_{\mathcal{J}} \geq 0$ holds element-wise, and $\mathbf{A}_{\mathcal{I} \cup \mathcal{K}} \geq \mathbf{A}_{\mathcal{J} \cup \mathcal{K}} \geq 0$ holds element-wise.

Since $\tilde{\mathbf{Q}}=(1-c)(\mathbf{I}-c \tilde{\mathbf{A}})^{-1}=(1-c) \sum_{j=0}^{\infty}(c \tilde{\mathbf{A}})^{j}$, we have

$$
\begin{aligned}
\mathrm{g}(s, t, \mathcal{I} \cup \mathcal{K})-\mathrm{g}(s, t, \mathcal{I}) & =(1-c) \sum_{j=0}^{\infty}\left(\left(c \mathbf{A}_{\mathcal{I}}\right)^{j}(t, s)-\left(c \mathbf{A}_{\mathcal{I} \cup \mathcal{K}}\right)^{j}(t, s)\right) \\
& \geq(1-c) \sum_{j=0}^{\infty}\left(\left(c \mathbf{A}_{\mathcal{J}}\right)^{j}(t, s)-\left(c \mathbf{A}_{\mathcal{J} \cup \mathcal{K}}\right)^{j}(t, s)\right)=\mathrm{g}(s, t, \mathcal{J} \cup \mathcal{K})-\mathrm{g}(s, t, \mathcal{J})
\end{aligned}
$$

Therefore, $\mathrm{g}(s, t, \mathcal{I})$ is sub-modular, which completes the proof of $\mathrm{P} 2$.

Proof of P4: Since $\mathrm{g}(\mathcal{S}, \mathcal{T}, \mathcal{I})=\sum_{s \in \mathcal{S}, t \in \mathcal{T}} \mathrm{g}(s, t, \mathcal{I})$ (In other words, $\mathrm{g}(\mathcal{S}, \mathcal{T}, \mathcal{I})$ is a non-negative linear combination of submodular functions), according to the linearity of sub-modular functions [19], we have that $\mathrm{g}(\mathcal{S}, \mathcal{T}, \mathcal{I})$ is also sub-modular, which completes the proof of $\mathrm{P} 4$.

Intuition. Here, we provide some intuition why $\mathrm{g}(s, t, \mathcal{I})$ and $\mathrm{g}(\mathcal{S}, \mathcal{T}, \mathcal{I})$ are sub-modular. According to Lemma 1 , for a given source $s$ and a given target $t, \mathrm{~g}(s, t, \mathcal{I} \cup \mathcal{K})-\mathrm{g}(s, t, \mathcal{I})$ accounts for the scores of all the paths from $s$ to $t$, which go through some nodes in $\mathcal{K}$ but none of the nodes in $\mathcal{I}$. Therefore, for a given set $\mathcal{K}$, if we already have a bigger subset $\mathcal{J}$, the additional benefit $(\mathrm{g}(s, t, \mathcal{J} \cup \mathcal{K})-\mathrm{g}(s, t, \mathcal{J}))$ will be relatively small, compared to the case where we have a smaller subset $\mathcal{I}$ $(\mathrm{g}(s, t, \mathcal{I} \cup \mathcal{K})-\mathrm{g}(s, t, \mathcal{I}))$. For example, in figure 1 , let $s=1, t=20$, and $\mathcal{I}=\{5\}, \mathcal{J}=\{2,5\}$. Then, if we have a new subset $\mathcal{K}=\{8\}$, the additional benefit for subset $\mathcal{I}$ accounts for all the paths from $s=1$ to $s=20$ which go through node 8 , but not node 5 (e.g., the green path, etc). While the additional benefit for subset $\mathcal{J}$ is 0 , since all the paths from $s=1$ to $t=20$ which go through node 8 must also go through some node in $\mathcal{J}$ (node 2 ).

\subsection{BASSET-N for Problem 1}

\subsubsection{BASSET-N Algorithm}

Our fast solution for Problem 1 is summarized in Alg. 1. In Alg. 1, after initialization (step 1), we first pick a node $i_{0}$ with the highest $\frac{\mathbf{r}(s, i) \mathbf{r}(i, t)}{\mathbf{r}(i, i)}$ (step 3). Then, in steps 4-14, we find the rest of the nodes in a greedy way. That is, in each outer loop, we try to find one more node while keeping the current $\mathcal{I}$ unchanged. According to P1 of theorem $1, \mathbf{v}(i)$ computed in step 7 is the gateway score for the subset $\mathcal{J}{ }^{3}$ If the current subset of nodes $\mathcal{I}$ can completely disconnect the source and the target (by setting them as sinks), we will stop the algorithm (step 12). Therefore, Alg. 1 always returns no more than $k$ nodes. It is worth pointing out that in Alg. 1, all the proximity scores are computed from the original graph $\mathbf{A}$. Therefore, we can utilize some powerful methods based on pre-computation to accelerate the whole process. To name a few, for a medium size graph $\mathbf{A}$ (e.g., a few thousands of nodes), we can pre-compute and store the matrix $\mathbf{Q}=(1-c)(\mathbf{I}-c \mathbf{A})^{-1}$; for large unipartite graphs and bipartite graphs, we can use the NB_LIN and BB_LIN algorithms, respectively [30].

\subsubsection{Analysis of BASSET-N.}

In this subsection, we analyze the effectiveness and the efficiency of Alg. 1. First, the effectiveness of the proposed BASSET-N is guaranteed by the following lemma. According to Lemma 2, although BASSET-N is a greedy algorithm, the results it outputs are near-optimal.

\footnotetext{
${ }^{3}$ This is because in random walk with restart, we have $\mathbf{r}(i, j)=\mathbf{Q}(j, i)$ for any $i, j[30]$.
} 


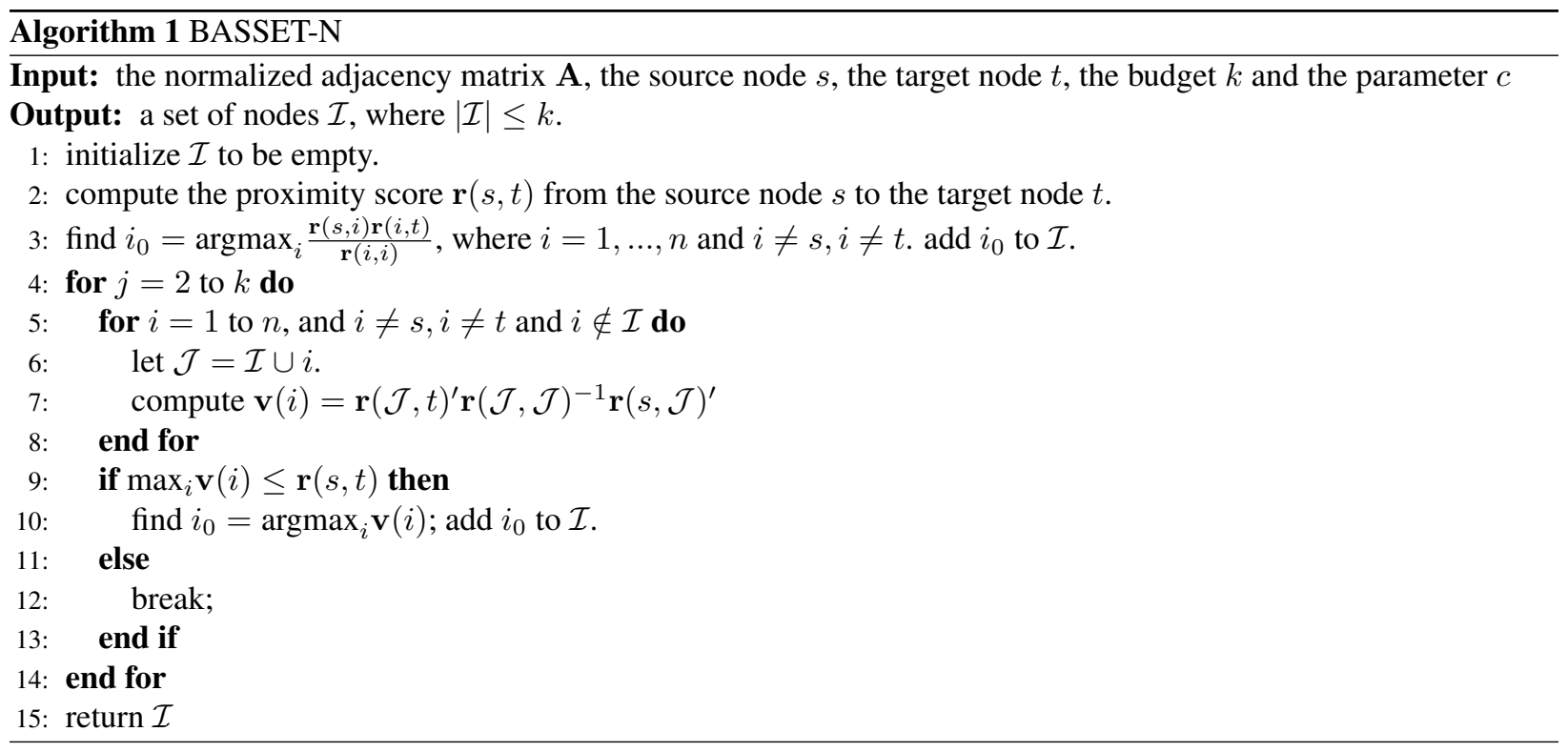

Lemma 2 Effectiveness of BASSET-N. Let $\mathcal{I}$ be the subset of nodes selected by Alg. 1 and $|\mathcal{I}|=k_{0} . \quad$ Then, $g(s, t, \mathcal{I}) \geq$ $(1-1 / e) \max _{|\mathcal{J}|=k_{0}} g(s, t, \mathcal{J})$, where $g(s, t, \mathcal{I})$, and $g(s, t, \mathcal{J})$ are defined by eq. $(1)$.

Proof: It is easy to verify that the node $i_{0}$ selected in step 10 of Alg. 1 satisfies $i_{0}=\operatorname{argmax}_{j \notin \mathcal{I}, j \neq s, j \neq t} \mathrm{~g}(s, t, \mathcal{I} \bigcup j)$. Also, we have $\mathrm{g}(s, t, \phi)=0$, where $\phi$ is an empty set. On the other hand, according to Theorem $1, \mathrm{~g}(s, t, \mathcal{I})$ is sub-modular wrt the subset $\mathcal{I}$. Therefore, we have $\mathrm{g}(s, t, \mathcal{I}) \geq(1-1 / e) \max _{|\mathcal{J}|=k_{0}} \mathrm{~g}(s, t, \mathcal{J})$, which completes the proof.

Next, we analyze the efficiency of BASSET-N, which is given in Lemma $3^{4}$. We can draw the following two conclusions, according to Lemma 3: (1) the proposed BASSET-N achieves a significant speedup over the straight-forward method $\left(O\left(n \cdot k^{4}\right) \mathrm{vs}\right.$. $O\left(\left(\begin{array}{l}n \\ k\end{array}\right) m\right)$ ). For example, in the graph with 100 nodes and 1,000 edges, in order to find the gateway with $k=5$ nodes, BASSET-N is more than 6 orders of magnitude faster, and the speedup quickly increases wrt the size of the graph; (2) the proposed BASSET-N is applicable to large graphs since it is linear wrt the number of the nodes.

Lemma 3 Efficiency of BASSET-N. The computational complexity of Alg. 1 is upper bounded by $O\left(n \cdot k^{4}\right)$.

Proof: The cost for steps 1-2 is constant. The cost for step 3 is $O(n)$. At each inner loop (steps 6-7), the cost is $O\left(n j^{3}+n j^{2}\right)$. The cost for steps 9-13 is $O(n)$. The outer loop has no more than $k-1$ iterations. Putting these together, the computational cost for BASSET-N is:

$$
\operatorname{Cost}(\text { BASSET-N }) \leq n+\sum_{j=1}^{k}\left(n j^{3}+n j^{2}+n\right)=n+n k+n \frac{k(k+1)(2 k+1)}{6}+n \frac{k^{2}(k+1)^{2}}{4}=O\left(n k^{4}\right)
$$

which completes the proof.

\subsection{BASSET-G for Problem 2}

\subsubsection{BASSET-G Algorithm}

Our fast solution for Problem 2 is summarized in Alg. 2. It works in a similar way as Alg. 1: after initialization (step 1), we first pick a node $i_{0}$ with the highest $\sum_{s \in \mathcal{S}, t \in \mathcal{T}} \frac{\mathbf{r}(s, i) \mathbf{r}(i, t)}{\mathbf{r}(i, i)}$ (step 3). Then, in steps 4-14, we find the rest of the nodes in a greedy way. That is, in each outer-loop, we try to find one more node while keeping the current $\mathcal{I}$ unchanged. If the current subset of the nodes $\mathcal{I}$ can completely disconnect the source group and the target group (by setting them as sinks), we will stop the algorithm (step 10). As in Alg. 1, all the proximity scores are computed from the original graph A. Therefore, we can again utilize those powerful pre-computation based methods to accelerate the whole process.

\footnotetext{
${ }^{4}$ Here, we assume that the cost to get one proximity score is constant, which can be achieved with pre-computation methods [30].
} 


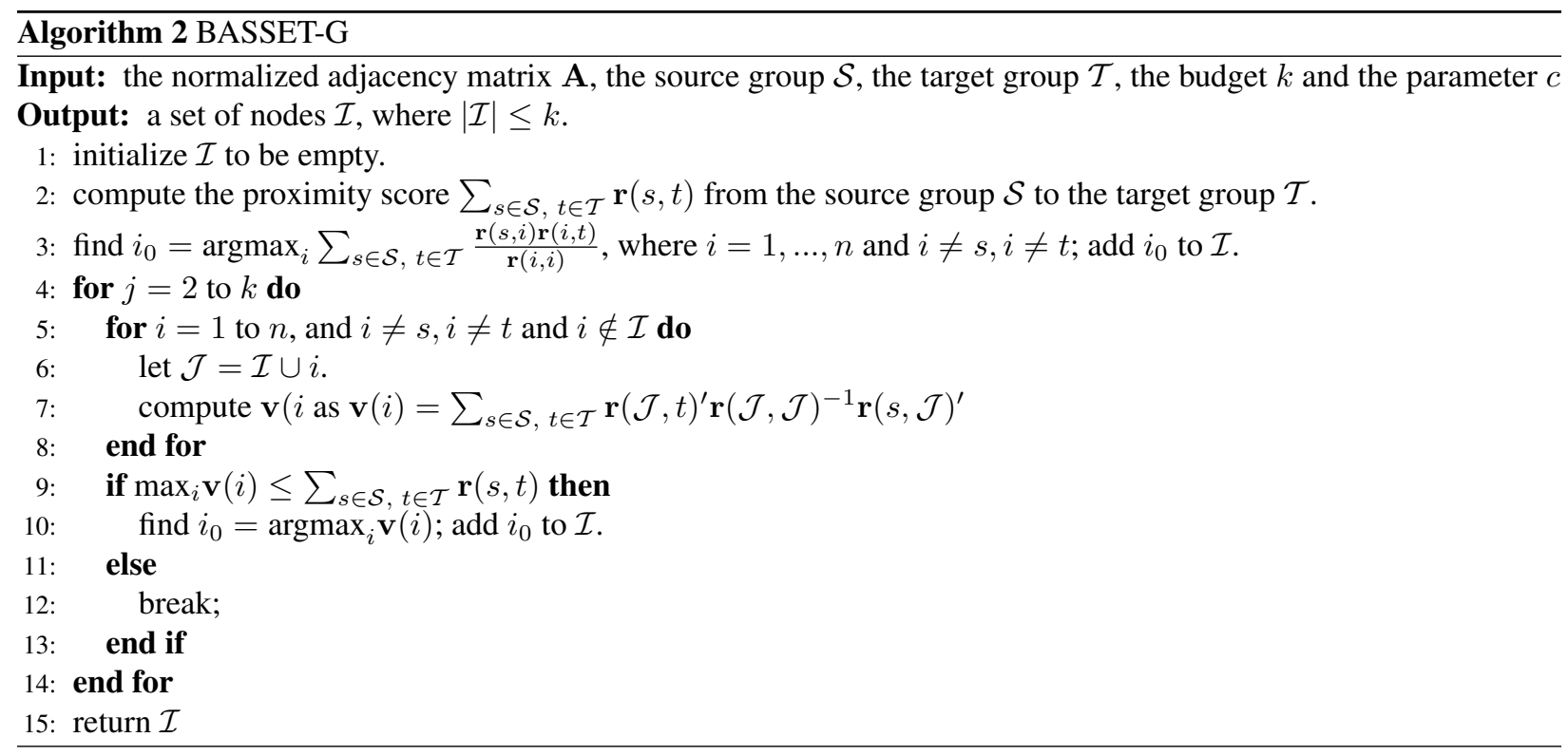

\subsubsection{Analysis of BASSET-G.}

The effectiveness and efficiency of the proposed BASSET-G are given in Lemma 4 and Lemma 5, respectively. Similar as BASSET$\mathrm{N}$, the proposed BASSET-G is (1) near-optimal; and (2) fast and scalable for large graphs.

Lemma 4 Effectiveness of BASSET-G. Let $\mathcal{I}$ be the subset of nodes selected by Alg. 2 and $|\mathcal{I}|=k_{0}$. Then, $g(\mathcal{S}, \mathcal{T}, \mathcal{I}) \geq$ $(1-1 / e) \max _{|\mathcal{J}|=k_{0}} g(\mathcal{S}, \mathcal{T}, \mathcal{J})$, where $g(\mathcal{S}, \mathcal{T}, \mathcal{I})$, and $g(\mathcal{S}, \mathcal{T}, \mathcal{J})$ are defined by eq. (4).

Proof: Similar as for Lemma 2. Omitted for brevity.

Lemma 5 Efficiency of BASSET-G. The computational complexity of Alg. 2 is upper bounded by $O\left(n \cdot(\max (k,|\mathcal{S}|,|\mathcal{T}|))^{4}\right)$.

Proof: Similar as for Lemma 3. Omitted for brevity.

\section{Experimental Evaluations}

In this section we present experimental results. All the experiments are designed to answer the following questions:

- Effectiveness: how effective are the proposed 'Gateway-ness' scores in real graphs?

- Efficiency: how fast and scalable are the proposed BASSET-N and BASSET-G?

\subsection{Experimental Setup}

Data sets. We used four real data sets, which are summarized in table 2.

Table 2: Summary of the data sets

\begin{tabular}{|c|c|c|c|}
\hline Name & $n$ & $m$ & Weight \\
\hline \hline Karate & 34 & 152 & No \\
\hline PolBooks & 105 & 882 & No \\
\hline$A A$ & 418,236 & $2,753,798$ & Yes \\
\hline NetFlix & $2,667,199$ & $56,919,190$ & No \\
\hline
\end{tabular}

The first data set (Karate) is an un-weighted unipartite graph, which describes friendship among the 34 members of a karate club at a US university [32]. Each node is a member in the karate club and the existence of the edge indicates that the two corresponding members are friends. Overall, we have $n=34$ nodes and $m=156$ edges. 
The second data set (PolBooks) is a co-purchasing book network. ${ }^{5}$ Each node is a political book and there is an edge between two books if purchased by the same person. Overall, we have $n=105$ nodes and $m=882$ edges.

The third data set $(A A)$ is a co-authorship network, where each node is an author and the edge weight is the number of the co-authored papers between the two corresponding persons. Overall, we have $n=418,236$ nodes and $m=2,753,798$ edges.

The last data set (NetFlix) is from the Netflix prize ${ }^{6}$. Rows represent users and columns represent movies. If a user has given a particular movie positive ratings ( 4 or 5), we connect them with an edge. In total, we have 2,667,199 nodes (2,649,429 users and 17,770 movies), and 56,919,190 edges.

Parameter settings and machine configurations. There is one parameter in BASSET-N and BASSET-G, the probability $c$ for random walk with restart. We set $c=0.95$, as suggested in [30]. For the computational cost, we report the wall-clock time. All the experiments ran on the same machine with four 2.4GHz AMD CPUs and 48GB memory, running Linux (2.6 kernel). For each experiment, we run it 10 times and report the average.

\subsection{Effectiveness}

Here, we evaluate the effectiveness of the proposed 'Gateway-ness' scores. We first compare with several candidate methods in terms of separating the source from the target. And then, we present various case studies. The basic idea of the proposed 'Gatewayness' scores is to find a subset of nodes which collectively play an important role in measuring the proximity from the source node (or source group) to the target node (or target group). Here, we want to validate this basic assumption. We compare it with the following alternative choices: (a) selecting $k$ nodes with the highest center-piece AND score (CePS-AND) [28]; (b) selecting $k$ nodes with the highest center-piece OR score (CePS-OR) [28]; (c) randomly selecting $k$ nodes (Rand); (d) randomly selecting $k$ nodes from the neighboring nodes of the source node and the target node (Neighbor-Rand); (e) selecting $k$ nodes with the highest $\frac{\mathbf{r}(s, i) \mathbf{r}(i, t)}{\mathbf{r}(i, i)}$ (Topk-Ind). We randomly select a source node $s$ and a target node $t,{ }^{7}$ and then use the different methods to select a subset $\mathcal{I}$ with $k$ nodes. Figure 2 presents the comparison results, where the $\mathrm{x}$-axis is the number of nodes selected $(k)$, and the $\mathrm{y}$-axis is the normalized decay in terms of the proximity score from the source node $s$ to the target node $t\left(\frac{\mathbf{r}(s, t)-\mathbf{r}_{\mathcal{I}}(s, t)}{\mathbf{r}(s, t)}\right)$. The resulting curves are averaged over 1,000 randomly chosen source-target pairs. From figure 2, we can see that (1) the proposed BASSET-N performs best in terms of separating the source from the target; (2)Topk-Ind, where we simply select $k$ nodes with highest $\frac{\mathbf{r}(s, i) \mathbf{r}(i, t)}{\mathbf{r}(i, i)}$, does not perform as well as BASSET-N, where we want to find a subset of $k$ nodes which collectively has the highest score $\mathbf{r}(\mathcal{I}, t)^{\prime} \mathbf{r}(\mathcal{I}, \mathcal{I})^{-1} \mathbf{r}(s, \mathcal{I})^{\prime}$.
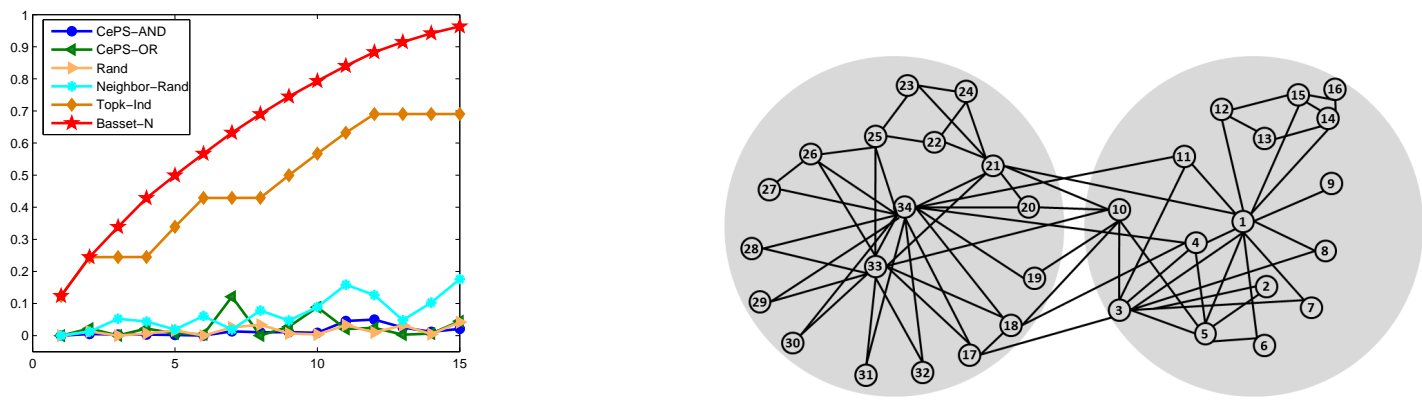

Figure 2: Effectiveness comparison between BASSET-N and alternatives. Normalized decay of proximity vs. $k$. Higher is better. The proposed BASSET-N (red star) is the best.

\subsubsection{Case Studies}

Next, we will show some case studies, to demonstrate the effectiveness of BASSET-N and BASSET-G.

Karate. We start with Karate graph, which is widely used in social network analysis. In figure 3, there are two different communities in the graph (shaded). In each community, there are some 'hub' nodes (e.g., nodes 33 and 34 in the left community; and nodes 1 and 4 in the right community). The two communities are connected by some 'bridging nodes' (e.g., nodes 3, 10, 19,

\footnotetext{
${ }^{5}$ http: //www. orgnet. com/

${ }^{6}$ http: //www. netflixprize.com/

${ }^{7}$ The result when source and target are a group of nodes is similar, and omitted for brevity.
} 
20). Table 3 presents the resulting gateways of BASSET-N with the budget $k=5$ for a few source-target pairs. The results are consistent with human intuition. The gateways either are the local center of the community that the source/target node belongs to, or are bridging nodes that connect the two communities when the source node and the target node belong to different communities. For example, if $s=1$ and $t=33$, the resulting nodes 3,10,11 are bridging nodes, while node 34 is the local center for the left community. Note that, we always return less than or equal to $k=5$ nodes. For example, if $s=15$ and $t=34$, we only output one node (node 1) as the gateway. This is because all the paths from node 15 to node 34 must go through node 1.

Table 3: BASSET-N on Karate graph

\begin{tabular}{|c|c|c|}
\hline Source $(s)$ & Target $(t)$ & Gateways $(\mathcal{I})$ \\
\hline \hline 24 & 31 & $\{33,34\}$ \\
\hline 15 & 34 & $\{1\}$ \\
\hline 1 & 33 & $\{3,10,11,21,34\}$ \\
\hline
\end{tabular}

Table 4: BASSET-N on PolBooks Graph. ('c' for 'conservative', 'l' for 'liberal', and 'n' for 'neutral')

\begin{tabular}{|c|c|c|}
\hline Node Index & Book Title & Label \\
\hline \hline 10 & Bush country & $\mathrm{c}$ \\
13 & Off with their heads & $\mathrm{c}$ \\
103 & Back up such up & $\mathrm{l}$ \\
5 & Sleeping with the devil & $\mathrm{n}$ \\
8 & Ghost wars & $\mathrm{n}$ \\
77 & Plan of attack & $\mathrm{n}$ \\
78 & Bush at war & $\mathrm{c}$ \\
59 & Rise of the vulcanes & $\mathrm{c}$ \\
52 & Allies & $\mathrm{c}$ \\
42 & The Bushes & $\mathrm{c}$ \\
\hline
\end{tabular}

PolBooks. For this data set, the nodes are political books and the existence of the edge indicates the co-purchasing (by the same person) of the two books. Each book is annotated by one of the following three labels: 'liberal', 'conservative' and 'neutral'. We pick a 'liberal' book ('The Price of Loyalty') as the source node, and a 'conservative' book ('Losing Bin Laden') as the target node. Then, we ran the proposed BASSET-N to find the gateway with 10 nodes. The result is presented in table 4 . The result is again consistent with human intuition, - the resulting gateway books are either popular books in one of the two communities ('conservative' vs. 'liberal') such as, 'Bush country' from 'conservative', 'Back up suck up' from 'liberal', etc; or those 'neutral' books which are likely to be purchased by readers from both communities (e.g., 'Sleeping with the devil', etc).

Table 5: BASSET-G on $A A$ Network.

\begin{tabular}{|c|c|c|}
\hline Source Group & Target Group & Gateway $(k=10)$ \\
\hline $\begin{array}{c}\text { Tom M. Mitchell, } \\
\text { William W. Cohen, } \\
\text { Jaime G. Carbonell }\end{array}$ & $\begin{array}{c}\text { David J. Dewitt, } \\
\text { Hector Garcia-Molina, } \\
\text { H. V. Jagadish }\end{array}$ & $\begin{array}{c}\text { Sunita Sarawagi, Christos Faloutsos, James P. Callan, } \\
\text { Rakesh Agrawal, Andrew Y. Ng, Yiming Yang, Rich Caruana, } \\
\text { Andrew Mccallum, Chengxiang Zhai, Sebastian Thrun, }\end{array}$ \\
\hline
\end{tabular}

(a) A group of people in 'text' to a group of people in 'databases'

\begin{tabular}{|c|c|c|}
\hline Source Group & Target Group & Gateway $(k=10)$ \\
\hline $\begin{array}{c}\text { Manuel Blum, } \\
\text { Christos H. Papadimitriou }\end{array}$ & $\begin{array}{c}\text { Vipin Kumar, Wei Wang, } \\
\text { George Karypis, } \\
\text { Mohammed J. Zaki }\end{array}$ & $\begin{array}{c}\text { Philip S. Yu, Mihalis Yannakakis, Hui Xiong, } \\
\text { Hongjun Lu, Sally A. Goldman, Jiawei Han, Moni Naor, } \\
\text { Mitsunori Ogihara, Richard M. Karp, Prabhakar Raghavan }\end{array}$ \\
\hline
\end{tabular}

(b) A group of people in 'theory ' to a group of people in 'bioinfomatics'

$A A$. We use this data set to perform case studies for the proposed BASSET-G. We choose (1) a group of people from a certain field (e.g., 'text', 'theory', etc) as the source group $\mathcal{S}$; and (2) another group of people in some other field (e.g., 'databases', 'bioinfomatics', etc) as the target group $\mathcal{T}$. Then, we ran the proposed BASSET-N to find the gateway with $k=10$ nodes. Table 5 lists some results. They are all consistent with human intuition, - the resulting authors are either productive authors in one of the two fields, or multi-disciplinary, who have close collaborations to both the source and the target groups of authors.

\subsection{Efficiency}

We will study the wall-clock running time of the proposed BASSET-N and BASSET-G here. Basically, we want to answer the following two questions:

1. (Speed) What is the speedup of the proposed BASSET-N and BASSET-G over the straightforward methods? 
2. (Scalability) How do BASSET-N and BASSET-G scale with the size of the graph ( $n$ and $m$ )?

First, we compare BASSET-N and BASSET-G with two straightforward methods: (1) 'Com-RWR', where we use combinatorial enumeration to find the gateway and, for each enumeration, we compute the proximity from the new graph; and (2) 'Com-Eval', where we use combinatorial enumeration to find the gateway, and for each enumeration, we compute the proximity from the original graph. Figure 4 shows the comparison on two real data sets. We can draw the following conclusions. (1) Straightforward methods ('Com-RWR' and 'Com-Eval') are computationally intractable even for a small graph. For example, on the Karate data set with only 34 nodes, it takes more than 20,560 seconds and 100, 000 seconds to find the $k=10$ gateway by 'Com-Eval' and by 'Com-RWR', respectively. (2) The speedup of the proposed BASSET-N and BASSET-G over both 'Com-Eval' and 'Com-RWR' is significant - in most cases, we achieve several (up to 6) orders of magnitude speedups. (3) The speedup of the proposed BASSET-N and BASSET-G over both 'Com-RWR' and 'Com-Eval' quickly increases wrt the size of the gateway $k$. Note that we stop running the program if it takes more than 100,000 seconds (i.e., longer than a day).

Next, we evaluate the scalability of the proposed BASSET-N and BASSET-G wrt the size of the graph, using the largest data set (NetFlix). From figure 5, we can make the following conclusions: (1) if we fix the number of nodes $(n)$ in the graph, the wall-clock time of both BASSET-N and BASSET-G is almost constant wrt the number of edges $(m)$; and $(2)$ if we fix the number of edges $(m)$ in the graph, the wall-clock time of both BASSET-N and BASSET-G is linear wrt the number of nodes $(n)$. Therefore, they are suitable for large graphs.
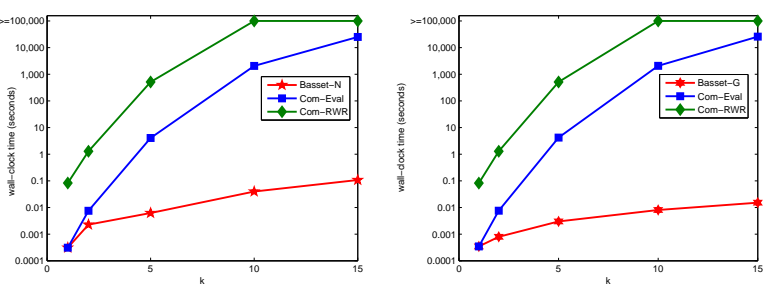

(a) Karate graph
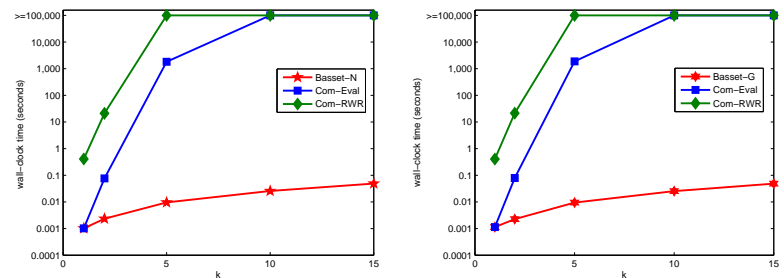

(b) PolBooks graph

Figure 4: Comparison of speed. Wall-clock time vs. $k$. Lower is better. Time is in logarithm scale. The proposed BASSET-N and BASSET-G (red star) are significantly faster.

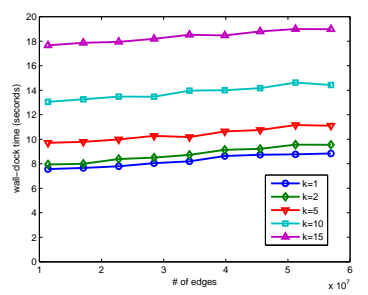

wall-clock time vs. $m$ (fix $n=2,667,199$ )

\section{(a) BASSET-N}

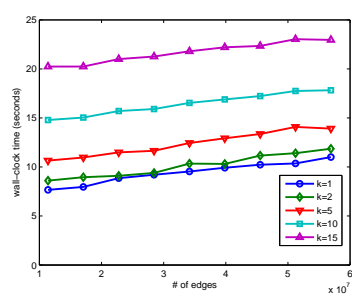

wall-clock time vs. $m$ (fix $n=2,667,199$ )

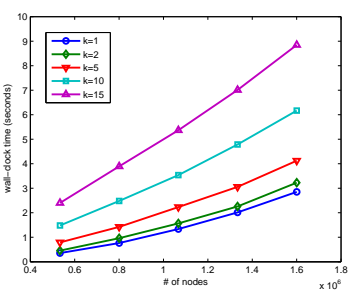

wall-clock time vs. $n$ (fix $m=23,874,700$ )

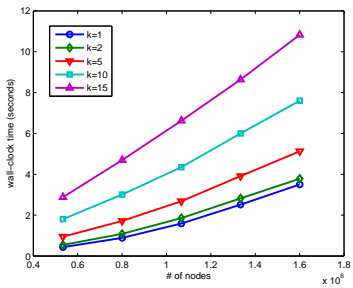

wall-clock time vs. $n$ (fix $m=23,874,700)$ (b) BASSET-G

Figure 5: Scalability of BASSET. Wall-clock time vs. the size of the graph. Lower is better. $|\mathcal{S}|=|\mathcal{T}|=5$.

\section{Related Work}

In this section, we review the related work, which can be categorized into four parts:

Betweenness centrality. The proposed 'Gateway-ness' scores relate to measures of betweenness centrality, both those based on the shortest path [10], as well as those based on random walk [22]. When the gateway set size is $k=1$, the proposed 'Gateway-ness' scores can be viewed as query-specific betweenness centrality measures. Moreover, in the proposed BASSET-N and BASSET-G, we aim to find a subset of nodes collectively, wherein traditional betweenness centrality, we usually calculate the score for each node independently (and then might pick $k$ nodes with the highest individual scores).

Connection subgraphs. In the proposed BASSET-N, the idea of finding a subset of nodes wrt the source/target is also related to the concept of connection subgraphs, such as $[9,18,28]$. However, in connection subgraphs, we aim to find a subset of nodes 
which have strong connections among themselves for the purpose of visualization. While in the proposed BASSET-N, we implicity encourage the resulting subset of nodes to be disconnected with each other so that they are able to collectively disconnect the target node from the source node to the largest extent (if we set them as sinks). It is interesting to notice that, if we want to find the gateway with $k=1$ for BASSET-N, it can be viewed as a normalized directed version of CePS-AND score [28]. ${ }^{8}$ Moreover, We allow the more general case where the source/target is a group of nodes in the proposed BASSET-G; however in connection subgraphs, the source/target is always a single node.

Graph proximity. The basic idea of the proposed BASSET-N and BASSET-G is to find a subset of nodes which will bring the largest decrease of the proximity score from the source node (or the source group) to the target node (or the target group). Graph proximity itself is an important building block in many graph mining settings. Representative work includes the BANKS system [1], link prediction [20], content-based image retrieval [14], cross-modal correlation discovery [24], pattern matching [29], ObjectRank [5], RelationalRank [11], etc.

Other related work in graph mining. In recent years, graph mining is a very hot research topic. Representative work includes pattern and law mining [3,6], frequent substructure discovery [31, 15], influence propagation [17], fraud and anomaly detection [21, 23], recommendation [8, 2], community mining and graph partition [16, 4, 12, 13, 27, 7], near-clique detection [25], etc.

\section{Conclusion}

In this paper, we study how to find good 'gateway' nodes in a graph, given one or more source and target nodes. Our main contributions are: (a) we formulate the problem precisely; (b) we develop BASSET-N and BASSET-G, two fast (up to 6,000,000x speedup) and scalable (linear wrt the number of the nodes in the graph) algorithms to solve it in a provably near-optimal fashion, using sub-modularity. We applied the proposed BASSET-N and BASSET-G on real data sets to validate the effectiveness and efficiency.

\section{Acknowledgements}

This material is based upon work supported by the National Science Foundation under Grants No. DBI-0640543 IIS-0705359 CNS-0721736 IIS0808661 iCAST and was performed under the auspices of the U.S. Department of Energy by Lawrence Livermore National Laboratory under contract No. DE-AC52-07NA27344. This work is also partially supported by an IBM Faculty Award, a SPRINT gift, with additional funding from Intel, and Hewlett-Packard. Any opinions, findings, and conclusions or recommendations expressed in this material are those of the author(s) and do not necessarily reflect the views of the National Science Foundation, or other funding parties.

\section{References}

[1] B. Aditya, G. Bhalotia, S. Chakrabarti, A. Hulgeri, C. Nakhe, and S. S. Parag. Banks: Browsing and keyword searching in relational databases. In $V L D B$, pages 1083-1086, 2002.

[2] D. Agarwal and S. Merugu. Predictive discrete latent factor models for large scale dyadic data. In KDD, pages 26-35, 2007.

[3] R. Albert, H. Jeong, and A.-L. Barabasi. Diameter of the world wide web. Nature, (401):130-131, 1999.

[4] L. Backstrom, D. P. Huttenlocher, J. M. Kleinberg, and X. Lan. Group formation in large social networks: membership, growth, and evolution. In KDD, pages 44-54, 2006.

[5] A. Balmin, V. Hristidis, and Y. Papakonstantinou. Objectrank: Authority-based keyword search in databases. In $V L D B$, pages 564-575, 2004.

[6] A. Broder, R. Kumar, F. Maghoul1, P. Raghavan, S. Rajagopalan, R. Stata, A. Tomkins, and J. Wiener. Graph structure in the web: experiments and models. In WWW Conf., 2000.

[7] J. Chen, O. R. Zaïane, and R. Goebel. Detecting communities in social networks using max-min modularity. In SDM, pages 978-989, 2009.

[8] H. Cheng, P.-N. Tan, J. Sticklen, and W. F. Punch. Recommendation via query centered random walk on k-partite graph. In ICDM, pages 457-462, 2007.

\footnotetext{
${ }^{8}$ To see this, notice that in the case $k=1$, in BASSET-N, we want to find the node with the highest $\frac{\mathbf{r}(s, i) \mathbf{r}(i, t)}{\mathbf{r}(i, i)}$; while in CePS-AND [28], it picks the nodes with the highest $\mathbf{r}(s, i) \mathbf{r}(t, i)$, where $i=1, \ldots, n$ and $i \neq s, i \neq t$.
} 
[9] C. Faloutsos, K. S. McCurley, and A. Tomkins. Fast discovery of connection subgraphs. In KDD, pages 118-127, 2004.

[10] L. C. Freeman. A set of measures of centrality based on betweenness. Sociometry, pages 35-41, 1977.

[11] F. Geerts, H. Mannila, and E. Terzi. Relational link-based ranking. In VLDB, pages 552-563, 2004.

[12] D. Gibson, J. Kleinberg, and P. Raghavan. Inferring web communities from link topology. In Ninth ACM Conference on Hypertext and Hypermedia, pages 225-234, New York, 1998.

[13] M. Girvan and M. E. J. Newman. Community structure is social and biological networks.

[14] J. He, M. Li, H.-J. Zhang, H. Tong, and C. Zhang. Manifold-ranking based image retrieval. In ACM Multimedia, pages 9-16, 2004.

[15] R. Jin, C. Wang, D. Polshakov, S. Parthasarathy, and G. Agrawal. Discovering frequent topological structures from graph datasets. In $K D D$, pages 606-611, 2005.

[16] G. Karypis and V. Kumar. Multilevel -way hypergraph partitioning. In DAC, pages 343-348, 1999.

[17] D. Kempe, J. Kleinberg, and E. Tardos. Maximizing the spread of influence through a social network. KDD, 2003.

[18] Y. Koren, S. C. North, and C. Volinsky. Measuring and extracting proximity in networks. In KDD, pages 245-255, 2006.

[19] A. Krause and C. Guestrin. Near-optimal nonmyopic value of information in graphical models. In UAI, pages 324-331, 2005.

[20] D. Liben-Nowell and J. Kleinberg. The link prediction problem for social networks. In Proc. CIKM, 2003.

[21] J. Neville, Ö. Simsek, D. Jensen, J. Komoroske, K. Palmer, and H. G. Goldberg. Using relational knowledge discovery to prevent securities fraud. In $K D D$, pages 449-458, 2005.

[22] M. Newman. A measure of betweenness centrality based on random walks. Social Networks, 27:39-54, 2005.

[23] C. C. Noble and D. J. Cook. Graph-based anomaly detection. In KDD, pages 631-636, 2003.

[24] J.-Y. Pan, H.-J. Yang, C. Faloutsos, and P. Duygulu. Automatic multimedia cross-modal correlation discovery. In KDD, pages 653-658, 2004.

[25] J. Pei, D. Jiang, and A. Zhang. On mining cross-graph quasi-cliques. In $K D D$, pages 228-238, 2005.

[26] W. Piegorsch and G. E. Casella. Inverting a sum of matrices. In SIAM Review, volume 32, pages 470-470, 1990.

[27] T. Qian, J. Srivastava, Z. Peng, and P. C.-Y. Sheu. Simultaneously finding fundamental articles and new topics using a community tracking method. In PAKDD, pages 796-803, 2009.

[28] H. Tong and C. Faloutsos. Center-piece subgraphs: problem definition and fast solutions. In KDD, pages 404-413, 2006.

[29] H. Tong, C. Faloutsos, B. Gallagher, and T. Eliassi-Rad. Fast best-effort pattern matching in large attributed graphs. In $K D D$, pages 737-746, 2007.

[30] H. Tong, C. Faloutsos, and J.-Y. Pan. Random walk with restart: Fast solutions and applications. Knowledge and Information Systems: An International Journal (KAIS), 2008.

[31] D. Xin, J. Han, X. Yan, and H. Cheng. Mining compressed frequent-pattern sets. In VLDB, pages 709-720, 2005.

[32] W. W. Zachary. An information flow model for conflict and fission in small groups. pages 452-473, 1977. 